\title{
Análisis de la composición de los tribunales de tesis doctorales españolas en Traducción e Interpretación
}

\author{
Cristina Rodríguez-Faneca*, Alexander Maz-Machado*, David Gutiérrez-Rubio* \\ *Universidad de Córdoba \\ I02rofac@uco.es $\div$ ORCID ID: https://orcid.org/0000-0002-0940-666X \\ ma1mamaa@uco.es $\div$ ORCID ID: https://orcid.org/0000-0002-4112-4363

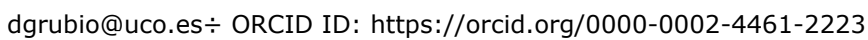

Recibido: 16-01-20; 2a versión: 20-07-20; Aceptado: 18-09-20; Publicado: 01-07-2021

Cómo citar este artículo/Citation: Rodríguez-Faneca, C.; Maz-Machado, A., Gutiérrez-Rubio, D. (2021). Análisis de la composición de los tribunales de tesis doctorales españolas en Traducción e Interpretación. Revista Española de Documentación Científica, 44 (3), e303. https://doi.org/ 10.3989/redc.2021.3.1750

Resumen: Se presenta un estudio sobre las tesis doctorales en Traducción e Interpretación en España, centrado en los directores y los tribunales encargados de su evaluación. Se consultó la base de datos de tesis doctorales españolas TESEO para identificar todas las tesis doctorales leídas durante el periodo 1997-2018, así como los participantes de los tribunales (presidente, secretario y vocales). Posteriormente se les identificó conforme a su género y se les asignó su filiación institucional para proceder a realizar los análisis pertinentes. Se hallaron cuatro directores con 10 o más tesis dirigidas y se identificó una tendencia a la dirección unitaria. Las universidades andaluzas, en su conjunto, son las que han producido el mayor número de tesis. Asímismo, se identificó a un grupo de profesores que ejercen gran influencia en los procesos de evaluación de las tesis, aunque no hayan dirigido un alto número tesis en el área.

Palabras clave: tesis doctorales; tribunales; traducción e interpretación; Bibliometría; patrones de producción científica; género.

\section{Analyzing the composition of Spanish Dissertation Committees in Translation and Inter- preting}

Abstract: A study about the Spanish Doctoral Dissertations in Translation and Interpreting subjets is presented, and it is aimed at the members of the Dissertation Committees and its advisors. The database TESEO was consulted in order to identify all the dissertations presented during the period 1997-2018, as well as the members of their Dissertation Committees (president, secretary and board members). Before analyzing the data available, their gender and their institutional affiliations were labeled. Four advisors have been in charge of more than ten doctoral dissertations. A tendency towards a single-advisor direction was also detected. The Andalusian universities have generated the greatest number of Doctoral Dissertations. It was also identified a group of professors who have influence over the evaluation of these doctoral dissertations, even though they have not been in charge of a high number of doctoral dissertations within this field.

Keywords: doctoral dissertations; dissertation committee; translation and interpreting; Bibliometrics; scientific production patterns; gender.

Copyright: (c) 2019 CSIC. Este es un artículo de acceso abierto distribuido bajo los términos de la licencia de uso y distribución Creative Commons Reconocimiento 4.0 Internacional (CC BY 4.0) 


\section{INTRODUCCIÓN}

Según lo establecido por la OCDE (1995), el proceso de formación de doctores constituye uno de los objetivos específicos que las universidades deben realizar con todas las garantías posibles y con la calidad necesaria. Hay que tener presente que los nuevos doctores serán los responsables de la investigación en el futuro $y$, en consecuencia, producirán un impacto tanto en el ámbito del desarrollo económico, como en los aspectos sociales

En España la formación de doctores ha sido regulada y modificada en varias ocasiones durante los últimos 20 años. En 1985 se implantó el concepto de programas de doctorado mediante el Real Decreto 185/1985 (BOE, 1985). En dicho documento se establece que deben superarse 32 créditos y obtener una suficiencia investigadora previa a la defensa de la tesis para obtener el título de doctor. Posteriormente, el Real Decreto 778/1998 (BOE, 1998) señalaba los requisitos de los estudios de posgrado y en concreto los de Tercer Ciclo conducentes a la obtención del título de doctor. Este Real Decreto (RD) establece la separación del doctorado en dos periodos; en primer lugar, un periodo de docencia que incluía cursar 20 créditos en asignaturas del programa de doctorado y, en segundo lugar, periodo de investigación para la realización de un trabajo de investigación. Esta fase concluía otorgando una suficiencia investigadora o un Diploma de Estudios Avanzados (DEA) que avalaban el inicio de la segunda fase con la realización de la tesis doctoral.

En los artículos 37 y 38 de la Ley Orgánica de Universidades (BOE, 2001) se hace referencia a los estudios de doctorado como el último ciclo de las enseñanzas oficiales universitarias. En el año 2007 se produce una reforma a través de la Ley orgánica 4/2007 (BOE, 2007) que consiste en una reestructuración de los estudios universitarios en ciclos de Grado, Máster y Doctorado. El Real Decreto 99/2011 (BOE, 2011) establece y regula las competencias que deben ser adquiridas por los doctorandos.

A través del Real Decreto 99/2011 se regulan las enseñanzas de doctorado en España. En este documento se destaca que "el doctorado debe jugar un papel fundamental como intersección entre el EEES y el EEI, ambos pilares fundamentales de la sociedad basada en el conocimiento" (BOE, 2011; p. 13909). Además, limitó la duración de los estudios de doctorado a tiempo completo a tres años como máximo desde la admisión hasta la defensa de la tesis. Posteriormente los RRDD 195/2016 (BOE, 2016) y 43/2015 (BOE, 2015) han modificado al primero de ellos (al RD 99/2011) y han incorpora- do algunos cambios de menor relevancia, como por ejemplo modificaciones en los criterios de acceso al programa de doctorado. Actualmente es posible acceder a los estudios de doctorado tanto por la posesión de un título de máster, DEA o suficiencia investigadora como por contar con una licenciatura o ingeniería que tenga un nivel equivalente al nivel de máster dentro del Marco Español de Cualificación para la Educación Superior (MECES).

La actual normativa (RD 99/2011, art. 13, p. 13918) dispone que: "La tesis doctoral consistirá en un trabajo original de investigación elaborado por el candidato en cualquier campo del conocimiento". En definitiva, los estudios de doctorado y las tesis doctorales resultantes son importantes a nivel social, académico y científico; por tanto, es valioso y de interés su estudio y análisis para identificar patrones y tendencias de distinto tipo.

Este estudio pretende analizar las redes invisibles de colaboración en la investigación en T\&I en España a partir del análisis de la composición de los tribunales de las tesis doctorales. Para ello, partimos de los registros de las tesis indexadas en la base de datos TESEO.

\section{ANTECEDENTES}

\subsection{Estudios bibliométricos sobre tesis doc- torales}

La elaboración de la tesis doctoral es el primer paso académico para constatar la capacidad de un investigador para diseñar y llevar a cabo una investigación científica en cualquier campo de las ciencias. Por ello es importante conocer y analizar todos los aspectos involucrados en dicho proceso.

En el ámbito internacional las tesis doctorales son un tema de estudio recurrente desde diversas perspectivas, tanto en ciencias como en ciencias sociales (Kagra y Sharma, 2014; Zong y otros, 2013). Entre ellas, existen investigaciones centradas en el papel del director de la tesis doctoral (Burnett, 1999; Wisker, 2012) o en la percepción que tienen los doctorandos respecto a los directores, (Kumar y otros, 2013), mientras que otras han abordado el género de los autores de las tesis (Seagram y otros, 1998). Asimismo, se ha investigado sobre los métodos de investigación empleados (Creswell y Miller, 1997) o la producción sobre un campo en un país determinado (Baggio y otros, 2014), entre otros estudios.

En España se han analizado las tesis doctorales producidas en instituciones españolas concretas (Maz-Machado y otros, 2012a), la producción de profesores de un área de conocimiento (Olivas-Ávila y Musi-Lechu- 
ga, 2010), una temática (Olmedilla y otros, 2017; Ramos-Pardo y Sánchez-Antolín, 2017), estudios sobre las mujeres (Torres-Ramírez y Torres-Salinas, 2005) o las tesis doctorales de doctorandos extranjeros en España (de Jesus Nascimento, 2000)

No cabe duda de que en el proceso de realización de una tesis doctoral confluyen una serie de aspectos que van más allá de la temática o del propio problema de investigación. Hay elementos curriculares, organizativos, de carácter institucional, y redes de tipo social relativas a la colaboración tanto entre directores como entre los miembros de los tribunales.

Asimismo, el papel de los tribunales que evalúan las tesis doctorales se ha analizado en diversas áreas del conocimiento. Por nombrar algunas: documentación y ciencias de la información (Olmeda-Gómez y otros, 2009), contabilidad (Casanueva-Roche y otros, 2007), educación matemática (Maz-Machado y otros, 2012b), ciencias del deporte (Ortega y otros, 2015) organización y gestión de empresas (Santos y otros, 2006), sociologia (Castelló, 2016) o cine (Repiso y otros, 2013).

\subsection{Estudios bibliométricos sobre Traducción e Interpretación}

En España, los estudios de Traducción e Interpretación (T\&I) se hallan implantados desde el año 1996 dentro del área de conocimiento "Traducción e Interpretación" (Comisión Académica del Consejo de Universidades, 1996). El largo Proceso de BoIonia dio como resultado la creación del Grado en Traducción e Interpretación en el año 2004.

A pesar de que durante toda dicha línea temporal se han venido realizando estudios de doctorado en T\&I, son pocos los estudios de tipo bibliométrico dentro del área de T\&I. Entre ellos destacamos el análisis de la producción científica de los de Traducción en España entre los años 1964 a 1994 (Pérez-Escudero, 2018). Este estudio se centró en la productividad por quinquenios, la productividad por tipología documental y en los idiomas de publicación, hallando asimismo cierta correlación entre el volumen de la producción respecto al propio establecimiento de la licenciatura de Traducción e Interpretación en España. Aixelá (2010) realizó una revisión bibliométrica acerca de la producción sobre Traducción e Interpretación médica recogida en la base de datos online Bibliografía de Interpretación y Traducción (BITRA), revisando el tipo de documentos, las lenguas de redacción y las distintas temáticas tratadas.

Otro trabajo de interés es el análisis de la base de datos Translation Studies Abstracts, en el cual se identificaron 27 categorías dentro de los estudios de Traducción e Interpretación (Zanettin y otros, 2105). A su vez, Valero-Garcés (2018) realizó un estudio sobre 200 tesis del Máster Universitario en Comunicación Intercultural, Interpretación y Traducción en los Servicios Públicos, en la combinación de idiomas chino-español, que se imparte en la Universidad de Alcalá de Madrid.

Así, logró identificar 7 dominios de investigación en T\&I: Aspectos culturales, situación del Servicio Público de Interpretación y Traducción, Materiales y herramientas, Aspectos terminológicos, Programas de Enseñanza, Interpretación y Traducción Comentada. Más recientemente, Pérez-Escudero (2018) realizó un estudio bibliométrico y webmétrico de la producción científica en Traducción Audiovisual, donde analizó la visibilidad e impacto del área en términos de citación. Estos estudios corroboran la actualidad e interés por conocer sobre qué y cómo se está realizando la investigación en temas relacionados con T\&I.

\section{MATERIALES Y MÉTODO}

Este trabajo presenta un estudio descriptivo Iongitudinal y retrospectivo de las tesis doctorales en T\&I defendidas en España hasta el año 2018. Como se halló que la lectura de la primera tesis fue en el año 1997, el periodo de análisis es el comprendido entre 1997 y 2018.

Los objetivos concretos que fueron propuestos para esta investigación son los siguientes:

1) Elaborar la serie temporal de tesis españolas indexadas en la base de datos TESEO relativa a la disciplina de Traducción e Interpretación desde 1997 hasta 2018.

2) Analizar la colaboración en la dirección de tesis doctorales sobre Traducción e Interpretación.

3) Analizar la composición de los tribunales de tesis doctorales en Traducción e Interpretación.

4) Realizar un análisis de género en relación con la dirección y participación en los tribunales de tesis en el área mencionada.

\subsection{Recopilación de la información}

Se realizó una búsqueda en la plataforma web de la base de datos TESEO para localizar los departamentos que, en cada centro, fuesen responsables de la dirección y producción de las tesis doctorales correspondientes. Teniendo en cuenta los registros de TESEO, se hallaron 7 departamentos específicos de Traducción e Interpretación (es decir, como área de conocimiento y departamento independiente, como es el caso de los departamentos 
de las universidades de Granada, Málaga y Salamanca, por nombrar algunos) y otros 9 departamentos que aglutinan dicha área de conocimiento junto con otras (como por ejemplo en el caso de los departamentos de las universidades Pablo de Olavide, Córdoba y Vigo). El total de departamentos consignados para este estudio es de 15, de las universidades de Granada, Málaga, Córdoba, Pablo de Olavide, Jaume I, Alicante, Salamanca, Pompeu Fabra, Autónoma de Barcelona, Pontificia Comillas, Vic, Vigo, País Vasco, San Jorge y de Murcia. En la figura 1 se presenta un ejemplo de cómo TESEO proporciona información relativa a cada tesis.

Para la descarga de la información se desarrolló una aplicación informática en Visual Basic que permitió automatizar la consulta de la información de todas las tesis doctorales. La búsqueda y descarga se realizó en el mes de febrero de 2019. Se importaron todos los campos que ofrece Teseo para cada tesis (título, autor, universidad, fecha, programa de doctorado, tribunal, descriptores y resumen)

Se hallaron 654 tesis doctorales producidas por los departamentos anteriormente listados y se realizó una revisión por dos expertos del área T\&I de la Universidad de Córdoba para verificar qué tesis correspondían con T\&I y cuáles a otras áreas afi- nes (por ej.: Lingüística, Terminología, Enseñanza de Lenguas, etc.). Durante la realización de este proceso se siguió el protocolo indicado por Fernández-Cano y otros (2008).

Al término de la selección y depuración realizada y de la posterior estandarización de las entradas halladas se obtuvieron un total de 389 tesis doctorales. Dichas tesis fueron leídas en los departamentos de Traducción e Interpretación de las universidades mencionadas entre los años 1997 y 2018, que conforman la población para este estudio.

\subsection{Análisis de la información}

Además de lo referido anteriormente, fue necesario estandarizar los nombres tanto de los directores como de los miembros de los tribunales, puesto que se hallaron dentro de la propia plataforma TESEO diversas denominaciones para una misma persona. Estos errores, propios de la base de datos TESEO, han sido señalados por otros investigadores previamente (Díaz-Campo, 2014; Repiso y otros, 2013). El objetivo de dicha estandarización es la de evitar entradas duplicadas haciendo referencia al mismo ente o informaciones equívocas, de cara tanto al posterior conteo como al procesa-

Figura 1. Ficha de tesis doctoral en TESEO

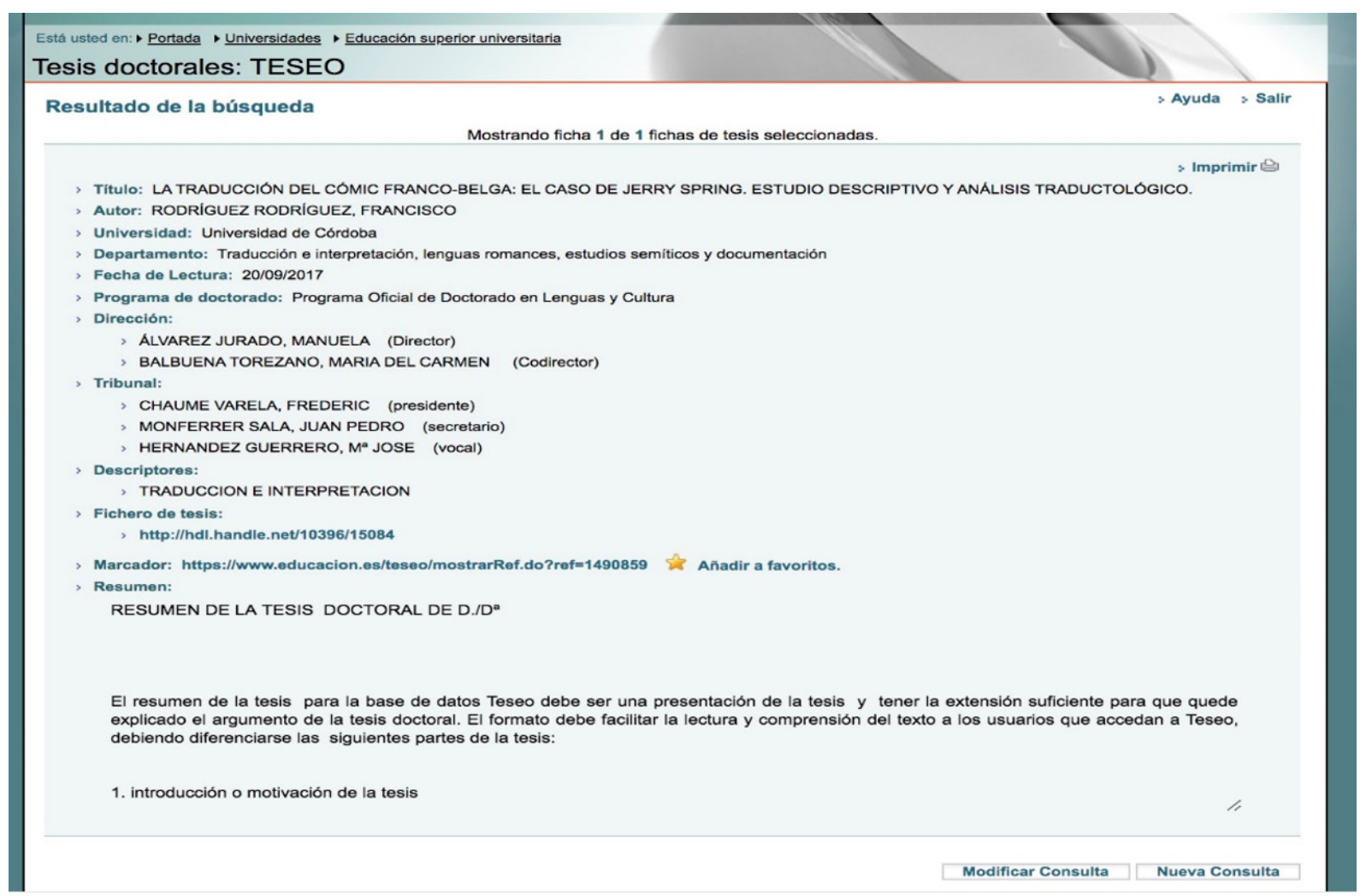


Figura 2. Proceso de selección de la muestra.

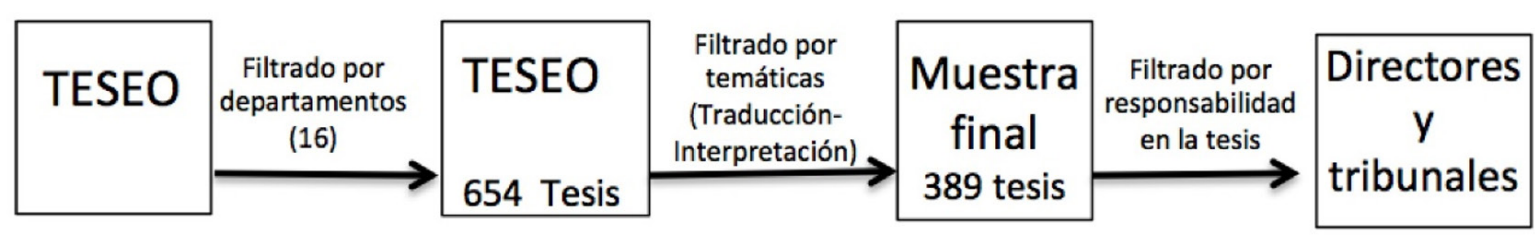

Figura 3. Producción diacrónica de tesis en T\&I.

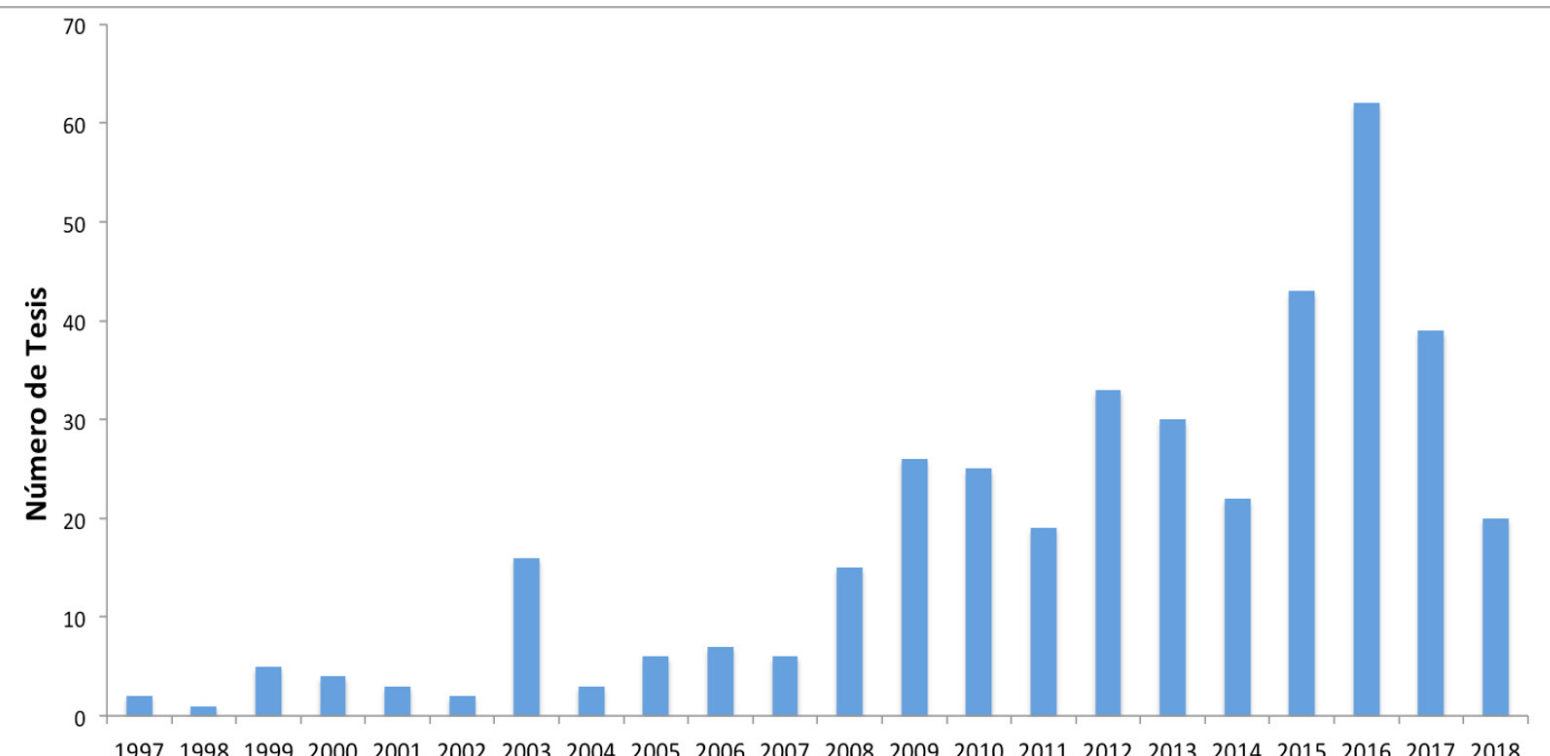

miento informático. El proceso de estandarización se realizó primero mediante una búsqueda difusa utilizando el módulo Fuzzy Lookup de la hoja de cálculo Excel para identificar nombres similares y una posterior etapa de depuración manual.

Posteriormente se procedió a realizar una búsqueda manual en las plataformas Google Scholar, Dialnet y Worldcat de cada director y miembro de tribunal, de manera que se averiguase su género y su afiliación profesional. La información se analizó con el software estadístico SPSS para obtener las tablas de frecuencias.

Cuando se habla de una Red, se entiende como un grupo de individuos o instituciones que de manera individual o agrupada establecen relaciones con otros para unos fines determinados (Velásquez y Aguilar, 2005). Para identificar las redes sociales de colaboración generadas a nivel de dirección, instituciones y participación en los tribunales se han construido varias matrices de adyacencia cuadradas sobre la coparticipación de profesores que han formado parte de 389 tribunales, los nombres de los profesores que han sido directores de tesis $y$, finalmente, una con los nombres de las instituciones a las que pertenecen los miembros de cada tribunal de tesis.

En cada matriz, cada columna y cada fila representa un profesor o institución que son, a su vez, los vértices de la red. La celda de intersección suma valores en función de las coincidencias en tribunales con otro profesor para la codirección y los tribunales. Para las instituciones se hereda la pertenencia a cada institución. La celda está vacía cuando no se coincide con otro miembro. Teniendo en cuenta que la coincidencia entre dos profesores o instituciones es recíproca, los vínculos que los unen no son dirigidos. Como ningún profesor coincide consigo mismo entonces, los vértices de la diagonal se representan con ceros.

\section{RESULTADOS}

La primera tesis identificada en T\&I dentro de este estudio es del año 1997. Se observa un incremento en la producción de tesis doctorales, si bien este aumento no es constante en el tiempo, sino que presenta fluctuaciones. Se alcanza la máxima 
Tabla I. Producción institucional de tesis doctorales en Traducción e Interpretación.

\begin{tabular}{|c|c|c|c|c|c|}
\hline Universidad & No de tesis & $\%$ & Comunidad Autónoma & Total & $\%$ \\
\hline Universidad de Granada & 81 & 20,8 & Andalucía & 180 & 46,3 \\
\hline Universidad de Málaga & 60 & 15,4 & & & \\
\hline Universidad de Córdoba & 25 & 6,4 & & & \\
\hline Universidad Pablo de Olavide & 14 & 3,6 & & & \\
\hline Universidad Jaume I de Castellón & 43 & 11,2 & Comunidad Valenciana & 77 & 19,8 \\
\hline Universidad de Alicante & 34 & 8,7 & & & \\
\hline Universidad de Salamanca & 48 & 12,3 & Castilla-León & 48 & 12,4 \\
\hline Universidad Pompeu Fabra & 27 & 6,9 & Cataluña & 36 & 9,2 \\
\hline Universidad Autónoma de Barcelona & 7 & 1,8 & & & \\
\hline $\begin{array}{l}\text { Universidad de Vic-Universidad Central de } \\
\text { Catalunya }\end{array}$ & 2 & 0,5 & & & \\
\hline Universidad de Vigo & 24 & 6,3 & Galicia & 24 & 6,2 \\
\hline Universidad Pontificia Comillas & 11 & 2,8 & Madrid & 11 & 2,8 \\
\hline Universidad del País Vasco & 9 & 2,3 & País Vasco & 9 & 2,3 \\
\hline Universidad de Murcia & 4 & 1,0 & Murcia & 4 & 1,0 \\
\hline Total & 389 & 100 & & 389 & 100 \\
\hline
\end{tabular}

producción en año 2016, seguida de la producción del año 2015, mientras que la menor producción se observa en 1998 (Figura 3).

La Universidad de Granada es la mayor productora de tesis doctorales en T\&I, seguida de la Universidad de Málaga. Entre las dos han producido el $36,2 \%$ del total de tesis que forman parte de este estudio. Si examinamos la distribución por comunidades autónomas, observamos que la mayor producción se da en las universidades de Andalucía, ya que las cuatro universidades que aportan tesis sobre T\&I producen el $46,3 \%$ de todas las

Tabla II. Directores de 4 o más tesis en T\&I (1997-2018)

\begin{tabular}{|l|l|l|l|}
\hline Director/a & No & Director/a & No \\
\hline Ortega Arjonilla, Emilio & 17 & García Peinado, M. Ángel & 5 \\
\hline Chaume Varela, Frederic & 13 & Kelly, Dorothy & 5 \\
\hline Vidal Claramonte, M. Carmen África & 13 & Marco Borillo, Josep & 5 \\
\hline Mogorrón Huerta, Pedro & 10 & Martin Ruano, M. Del Rosario & 5 \\
\hline Zaro Vera, Juan Jesús & 9 & Brumme, Jenny Elise & 4 \\
\hline Corpas Pastor, Gloria & 8 & Carbonell Cortes, Ovidio & 4 \\
\hline Faber Benítez, Pamela & 8 & Elena García, María Pilar & 4 \\
\hline Collados Ais, Ángela & 7 & García Izquierdo, Isabel & 4 \\
\hline García Calderón, Ángeles & 7 & García Palacios, Joaquín & 4 \\
\hline Jiménez Hurtado, Catalina & 7 & Guzmán Tirado, Rafael & 4 \\
\hline Way Catherine, Louise & 7 & Mata Pastor, Carmen & 4 \\
\hline Álvarez Jurado, Manuela & 6 & Mayoral Asensio, Roberto & 4 \\
\hline Balbuena Torezano, M. Del Carmen & 6 & Muñoz Martín, Ricardo & 4 \\
\hline Merino Álvarez, Raquel & 6 & Navarro Domínguez, Fernando & 4 \\
\hline Roser Nebot, Nicolás & 6 & Padilla Benítez, Presentación & 4 \\
\hline Toda Iglesia, Fernando & 6 & Sabio Pinilla, José Antonio & 4 \\
\hline Borja Albi, Anabel & 5 & Sales Salvador, Adoración & 4 \\
\hline Franco Aixelá, Javier & 5 & Tercedor Sánchez, M. Isabel & 4 \\
\hline Fuentes Luque, Adrián & 5 & Vega Cernuda, Miguel Ángel & 4 \\
\hline
\end{tabular}


tesis analizadas, seguidas de las dos universidades valencianas con el 19,8\% (Tabla I).

En la responsabilidad de la dirección de las tesis doctorales en T\&I en España han participado 244 profesores. El profesor Emilio Ortega de la Universidad de Málaga es quien ha dirigido el mayor número de tesis en T\&I con 17, seguido por Frederic Chaume de la Universidad Jaume I y Carmen África Vidal de la Universidad de Salamanca, con 13 tesis doctorales de T\&I cada uno (Tabla II). Se observó, además, que 31 doctorandos pasaron de ser autores de tesis doctorales a ser directores; de ellos, 20 son mujeres y 11 hombres. Esto es una cantidad muy pequeña, puesto que no alcanza a representar siquiera el $10 \%$ del total de doctorandos.
Teniendo en cuenta los datos presentados, se observa que la dirección de tesis en T\&I en España no presenta un sesgo de género, puesto que los porcentajes de hombres y mujeres son muy cercanos entre sí (Tabla III). Si bien las mujeres son mayoría respecto a los hombres en un $5 \%$, lo cual no es un valor significativo.

Tabla III. Género en la dirección de tesis doctorales en T\&I.

\begin{tabular}{|l|l|l|}
\hline Género & N & $\%$ \\
\hline H & 251 & 47,4 \\
\hline M & 278 & 52,6 \\
\hline Total & 529 & 100,0 \\
\hline
\end{tabular}

Colaboración en los tribunales de tesis doctorales

Figura 4. Red de colaboración institucional en la dirección de tesis doctorales en T\&I con participación en 5 o más tesis.

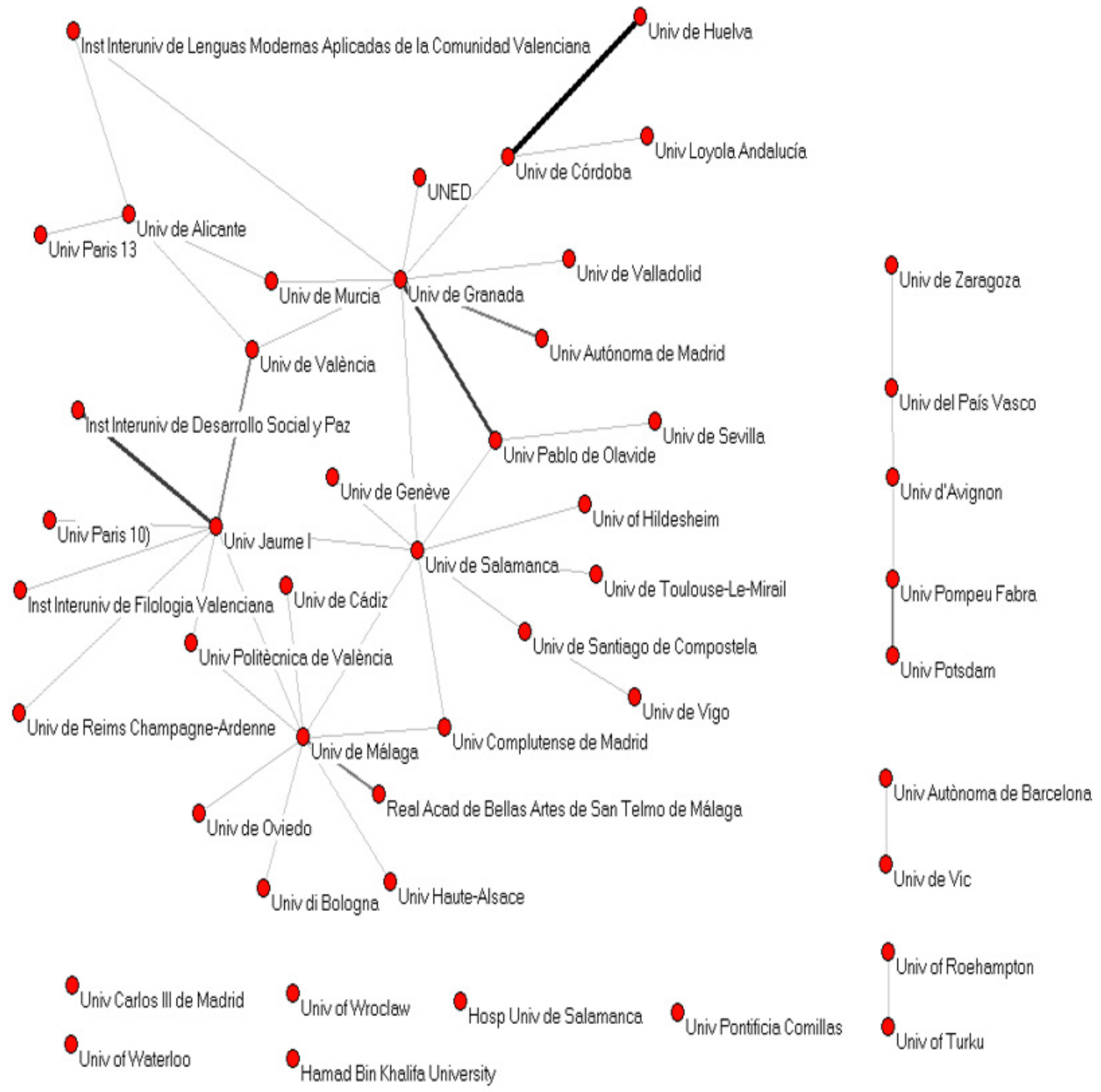


En la conformación de los tribunales encargados de evaluar las 389 tesis participaron profesores o profesionales de 175 instituciones españolas y extranjeras distintas (universidades, centros de investigación, entes administrativos dependientes de comunidades autónomas, institutos privados, hospitales, etc.); estos entes que aparecen en el grafo representan los nodos de la red (Figura 4). El grado de centralidad de la red de colaboración institucional es bajo, un $4,17 \%$, lo que indica una gran dispersión debido a la participación de miembros de muchos centros o universidades.

No obstante, se observa una gran colaboración entre las universidades de Córdoba-Huelva, Granada-Pablo de Olavide, Universidad Jaume I-Instituto interuniversitario de Desarrollo Social y Paz (Figura 4). Un hecho destacable sería que la codirección de las tesis de la Universidad Pompeu Fabra se realiza muy frecuentemente con algunas universidades extranjeras (Universidad de Potsdam y Universidad de Avignon). También se hallaron nódulos de codirección entre universidades extranjeras: University of Roehampton y University of Turku.

En la tabla IV se presentan las 10 instituciones con mayor representación entre los miembros de tribunales de las tesis doctorales de T\&I. Se observa que la UGR es la universidad que ha participado en el mayor número de tribunales, con un $40,4 \%$. En estos tribunales también participaron 207 investigadores de 115 universidades extranjeras. La mayor presencia la ostenta el University College London (UCL) con 23 profesores, seguida de la Université de Genève, University of Vienna y la University of Wolverhampton, con 7 profesores cada una.
Tabla IV. Instituciones con mayor participación en tribunales de tesis.

\begin{tabular}{|l|l|l|}
\hline Institución & Frecuencia & \% \\
\hline Universidad de Granada & 157 & 40,4 \\
\hline Universidad de Málaga & 116 & 29,8 \\
\hline Universidad de Salamanca & 99 & 25,4 \\
\hline Universitat Jaume I & 67 & 17,2 \\
\hline Universidad de Córdoba & 49 & 12,6 \\
\hline Universidad de Alicante & 41 & 10,5 \\
\hline $\begin{array}{l}\text { Universitat Autònoma de } \\
\text { Barcelona }\end{array}$ & 41 & 10,5 \\
\hline Universidad Pablo de Olavide & 41 & 10,5 \\
\hline Universidade de Vigo & 40 & 10,3 \\
\hline Universidad Pompeu Fabra & 38 & 9,8 \\
\hline
\end{tabular}

Existe una fuerte correlación positiva y significativa $(\rho=0,895)$ entre el número de tesis leídas en una universidad y el número de participantes en tribunales por parte de dicha universidad, si bien debe tenerse en cuenta que la mayoría de universidades exigen la presencia de un miembro de la propia universidad en el tribunal.

En total, 1618 personas formaron parte de los tribunales que evaluaron las tesis doctorales. El $50,4 \%$ de ellas fueron mujeres y el $49,6 \%$ hombres, con lo que se observa una paridad casi simétrica. Sin embargo, el sesgo de género surge al analizar quién desempeña el rol de presidente del tribunal; así, en el 60,2\% de las ocasiones lo ejercieron hombres, frente al $39,8 \%$ de ocasiones donde la presidencia fue ostentada por una mujer. La situación se invierte en el rol de secretario, pues

Figura 5. Composición de los tribunales de tesis en T\&I según el género.

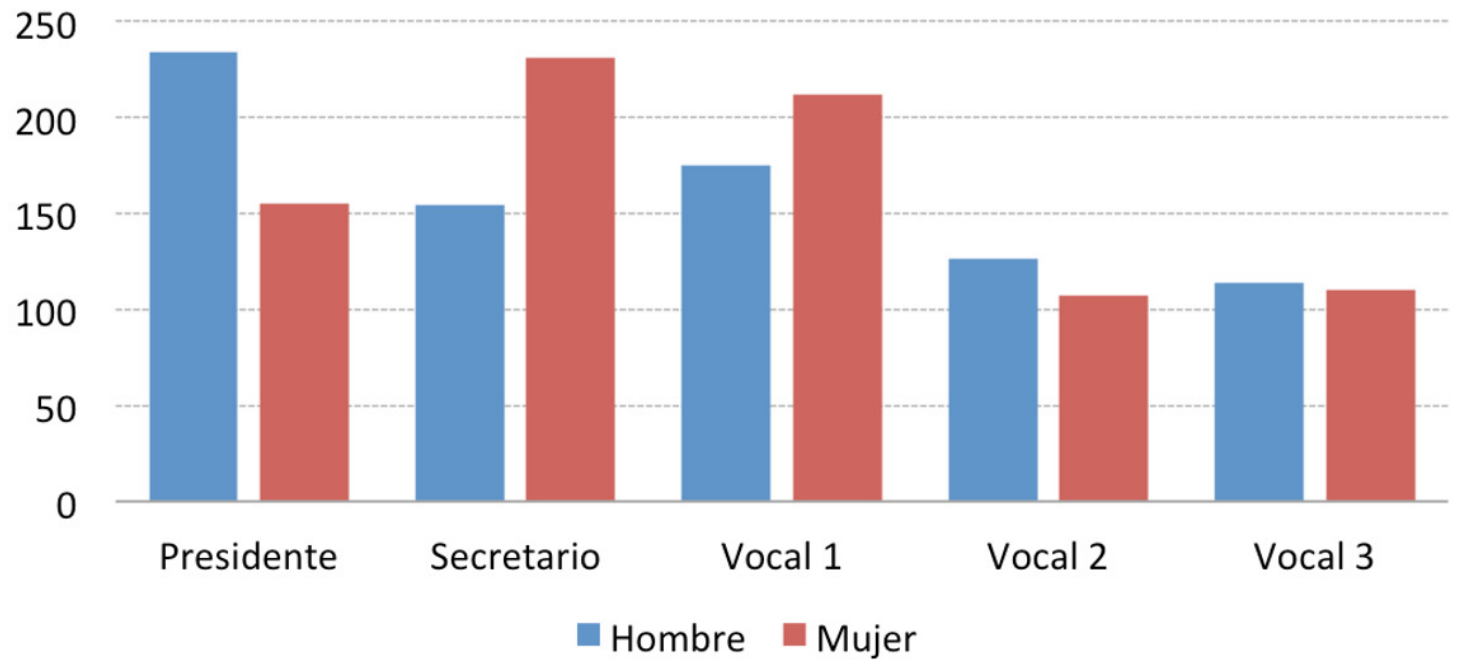


en este caso las mujeres ejercieron este rol un $59,38 \%$ de las ocasiones, mientras que los hombres el 39,59\% de las veces (Figura 5).

Para contrastar si la distribución de los cargos en el tribunal era independiente del género, se realizó el contraste chi-cuadrado de independencia. Se obtuvo un $p$-valor de 0,000 lo que indica que las diferencias observadas son estadísticamente significativas y no están enteramente producidas por el azar.

Desde un punto de vista descriptivo, en las tesis con un único director se observa una tendencia que el presidente del tribunal sea de género masculino cuando el director también lo es (casi el doble de probabilidad), mientras que cuando la directora es de género femenino, se aprecia igualdad en el género del presidente (Tabla V). Para comprobar si esas diferencias son significativas o fruto del azar se realizó el test exacto de Fisher, obteniendo un p-valor de 0,0084 . Es decir, existe una tendencia, más allá del azar, de elegir un presidente hombre cuando el director de la tesis también es hombre, a un nivel $a=0,05$.

Para las tesis en codirección, se aprecia una mayor tendencia a elegir un presidente de género masculino cuando al menos uno de los codirectores también lo sea. El test chi-cuadrado de independencia arroja un p-valor de 0,0604, que no evidenciaría diferencias estadísticamente significativas a un nivel $a=0,05$.

En los tribunales de las tesis doctorales de T\&I han participado 1618 profesores o profesionales españoles y extranjeros. Un grupo de 20 profesores han participado en más de 10 tribunales de tesis. Destacan Juan Jesús Zaro y África Vidal, con 24 y 23 participaciones, respectivamente (Tabla VI).

Si analizamos la ratio del número de tribunales en los que un profesor ha participado frente al total como tribunal o como director, es posible en cierta medida identificar a aquellos profesores que han tenido la mayor influencia en los procesos conducentes a la formación de los nuevos doctores en T\&I en España durante el periodo mencionado. Así, se observó que los profesores que podrían haber tenido mayor influencia son Marcos Rodríguez y Ana Belén Martínez (Tabla VII). Se hace patente que la influencia se ejerce más por participar en tribunales que por dirigir tesis. El director con mayor número de tesis dirigidas no alcanza a tener una influencia igual a 1 , mientras hay dos profesores que sin haber dirigido ninguna tesis en T\&I, al menos en las que tienen cabida en el presente estudio, han estado presentes en los tribunales de 19 tesis, es decir, casi en el 5\% del total.

Tabla V. Género del presidente del tribunal de tesis según género del director(es).

\begin{tabular}{|l|l|l|l|l|l|l|}
\hline Director & Género director & \multicolumn{5}{|c|}{ Género presidente } \\
\hline & & Hombre & \% & Mujer & \% & Total \\
\hline Único & Hombre & 93 & 23,9 & 49 & 12,6 & 142 \\
\hline & Mujer & 58 & 14,9 & 60 & 15,4 & 118 \\
\hline Codirigido & Hombre-Mujer & 45 & 11,6 & 15 & 3,9 & 60 \\
\hline & Mujer-Mujer & 26 & 6,7 & 22 & 5,7 & 48 \\
\hline & Hombre-Hombre & 12 & 3,1 & 9 & 2,3 & 21 \\
\hline Total & $\mathbf{2 3 4}$ & $\mathbf{6 0 , 2}$ & $\mathbf{1 5 5}$ & $\mathbf{3 9 , 8}$ & $\mathbf{3 8 9}$ & \\
\hline
\end{tabular}

Tabla VI. Profesores con 10 o más participaciones en tribunales de T\&I en el periodo 1997-2018.

\begin{tabular}{|c|c|c|c|}
\hline Miembro de tribunal & No de trib. & Miembro de tribunal & No de trib. \\
\hline Zaro Vera, Juan Jesús & 24 & Mayoral Asensio, Roberto & 12 \\
\hline Vidal Claramonte, M. C. África & 23 & Marco Borillo, Josep & 11 \\
\hline Campos Plaza, Nicolas Antonio & 19 & Sales Salvador, Adoración & 11 \\
\hline Chaume Varela, Frederic & 19 & Tercedor Sánchez, M. Isabel & 11 \\
\hline Díaz Cintas, Jorge & 19 & Ahumada Lara, Ignacio & 10 \\
\hline Faber Benítez, Pamela & 14 & Carbonell Cortes, Ovidio & 10 \\
\hline Ortega Arjonilla, Emilio & 14 & Elena García, María Pilar & 10 \\
\hline Jiménez Hurtado, Catalina & 13 & Fernández Nistal, Purificación & 10 \\
\hline Martin Ruano, M. Del Rosario & 13 & Rodríguez Espinosa, Marcos & 10 \\
\hline Navarro Domínguez, Fernando & 13 & Toda Iglesia, Fernando & 10 \\
\hline
\end{tabular}


Tabla VII. Profesores con mayor incidencia en las tesis de T\&I.

\begin{tabular}{|c|c|c|c|c|c|c|}
\hline $\mathbf{N}$ & Nombre & Tribunales (A) & Tesis dirigidas (B) & $A+B$ & \% de 389 & A/B \\
\hline 1 & Rodríguez, Marcos & 10 & 1 & 11 & 2,83 & 10 \\
\hline 2 & Martínez, Ana Belén & 9 & 1 & 10 & 2,57 & 9 \\
\hline 3 & Baigorri, Jesús & 9 & 2 & 11 & 2,83 & 4,5 \\
\hline 4 & Navarro, Fernando & 13 & 3 & 16 & 4,11 & 4,3 \\
\hline 5 & Mayoral, Roberto & 12 & 3 & 15 & 3,86 & 4 \\
\hline 6 & Mata, Carmen & 8 & 2 & 10 & 2,57 & 4 \\
\hline 7 & Sales, Adoración & 11 & 3 & 14 & 3,6 & 3,7 \\
\hline 8 & Martin, Ma Del Rosario & 13 & 4 & 17 & 4,37 & 3,3 \\
\hline 9 & Elena, María Pilar & 10 & 3 & 13 & 3,34 & 3,3 \\
\hline 10 & Zaro, Juan Jesús & 24 & 8 & 32 & 8,23 & 3 \\
\hline 11 & García, Miguel Ángel & 9 & 3 & 12 & 3,08 & 3 \\
\hline 12 & Marco, Josep & 11 & 4 & 15 & 3,86 & 2,8 \\
\hline 13 & Tercedor, M. Isabel & 11 & 4 & 15 & 3,86 & 2,8 \\
\hline 14 & Fernández, M. Manuela & 8 & 3 & 11 & 2,83 & 2,7 \\
\hline 15 & Fuentes, Adrián & 8 & 3 & 11 & 2,83 & 2,7 \\
\hline 16 & Carbonell, Ovidio & 10 & 4 & 14 & 3,6 & 2,5 \\
\hline 17 & Jiménez, Catalina & 13 & 6 & 19 & 4,88 & 2,2 \\
\hline 18 & Faber, Pamela & 14 & 7 & 21 & 5,4 & 2 \\
\hline 19 & Toda, Fernando & 10 & 5 & 15 & 3,86 & 2 \\
\hline 20 & Vidal, M. C. África & 23 & 12 & 35 & 9 & 1,9 \\
\hline 21 & Chaume, Frederic & 19 & 11 & 30 & 7,71 & 1,7 \\
\hline 22 & Padilla, Presentación & 6 & 4 & 10 & 2,57 & 1,5 \\
\hline 23 & Way, Louise & 8 & 6 & 14 & 3,6 & 1,3 \\
\hline 24 & Collados, Ángela & 6 & 6 & 12 & 3,08 & 1 \\
\hline 25 & Roser, Nicolás & 6 & 6 & 12 & 3,08 & 1 \\
\hline 26 & Franco, Javier & 5 & 5 & 10 & 2,57 & 1 \\
\hline 27 & Kelly, Dorothy & 5 & 5 & 10 & 2,57 & 1 \\
\hline 28 & Ortega, Emilio & 14 & 17 & 31 & 7,96 & 0,8 \\
\hline 29 & Mogorrón, Pedro & 5 & 10 & 15 & 3,86 & 0,5 \\
\hline 30 & Corpas, Gloria & 3 & 7 & 10 & 2,57 & 0,4 \\
\hline 31 & Campos, Nicolás A. & 19 & 0 & 19 & 4,88 & - \\
\hline 32 & Díaz, Jorge & 19 & 0 & 19 & 4,88 & - \\
\hline
\end{tabular}

\section{DISCUSIÓN Y CONCLUSIONES}

El presente estudio identificó 389 tesis doctorales sobre T\&I defendidas entre los años 1997 y 2018 en 16 departamentos de universidades españolas que incorporan el área de Traducción e Interpretación. Los resultados revelan una serie de patrones que poseen similitudes con los obtenidos en otros estudios sobre tesis doctorales en diferentes áreas del conocimiento en España.

La producción de tesis doctorales en el área de T\&I ha experimentado un aumento hasta el año 2016 pero en los dos últimos años se evidencia un decrecimiento. Las universidades de Granada y
Málaga lideran la producción de tesis doctorales en T\&I.

En cuanto al número, se observa que el aumento de tesis realizadas en el periodo es semejante a las tendencias generales que se vienen dando en España. Sin embargo, el promedio de tesis por año es alto, con 18,52 tesis doctorales al año, y superior al de otros campos de las Ciencias Sociales, como por ejemplo, las 13,77 tesis doctorales en el área de turismo (Peña Sánchez y otros, 2019), el 13,03 sobre televisión (Repiso y otros, 2011), el 8,11 en bibliometría (López-Cózar y otros, 2006) o 3 en moda (Alarcón, 2019). 
Se ha confirmado que la dirección de las tesis doctorales analizadas no presenta sesgo de género, mientras que en la figura de presidente del tribunal sí parece haber tendencia a escoger más a hombres que a mujeres. En la dirección de las tesis se observan porcentajes casi de equilibrio entre hombres $(47,4 \%)$ y mujeres $(52,6 \%)$, algo que no sucede en otros campos donde los directores hombres presentan grandes diferencias respecto a a las mujeres, como enfermería, donde alcanzan el 74,20 \% (do Nascimento y Pérez, 2011), en ciencia y tecnología, con el $72,5 \%$ (Mosteiro y Porto, 2014) o en las tesis doctorales sobre análisis bibliométrico en psicología, con el $73,33 \%$ (Osca-Lluch y otros, 2013).

Se ha constatado la existencia de cuatro directores de tesis doctorales con cierta influencia, pues acumulan más de 10 tesis dirigidas que pertenecen a las universidades de Alicante, Jaume I, Málaga y Salamanca. El $66,83 \%$ de las tesis han sido dirigidas por una sola persona, mientras que el $33,17 \%$ han tenido más de un director a su cargo.

Price (1973) acuñó el término de colegio invisible para designar a ciertos grupos de científicos que intercambian información sobre temas similares. En ocasiones estos colegios funcionan como redes de poder; en el caso de las tesis doctorales un reducido número de investigadores tienen el control de lo que se investiga y de quiénes lo hacen. Por ende, estos colegios pueden condicionar las decisiones científicas en un campo determinado (Casanueva-Roche y otros, 2007). En este estudio, el colegio invisible se evidencia por la influencia de algunos investigadores pese a tener solo una o dos tesis doctorales dirigidas ( $y$, en otros casos, ninguna). Se ha constatado que existe un grupo de profesores que ejercen un papel de colegio invisible que influye en valoración de las tesis de T\&I. Este patrón no es exclusivo de T\&I sino que también es patente en otras áreas, como los tribunales de las tesis de educación matemática (Maz-Machado y otros, 2012a).

En cuanto a la presidencia de los tribunales que evalúan las tesis doctorales se evidencia la preferencia de elegir a un hombre sobre una mujer para este papel, donde los hombres representan el $60,2 \%$, frente al 39,8\% de las mujeres. Si bien hay un sesgo en contra de las mujeres, este es menor que el hallado para las tesis doctorales de otras áreas como en ciencias del deporte, donde las mujeres que ostentaron la presidencia del tribunal solo alcanzó el $12 \%$ (Ortega y otros, 2015), mismo porcentaje que en Ciencia y Tecnologia (Mosteiro y Porto, 2014) o Psicología del Deporte, con solamente el $15,25 \%$.
Globalmente, en la composición de los tribunales se observa un equilibrio en cuanto al género; el $49,6 \%$ de los participantes son hombres, frente al $50,4 \%$ de mujeres. Esto difiere de los hallazgos relativos a otras áreas o temáticas, como en enfermería, donde las mujeres solo representan el $26,7 \%$ de los miembros del tribunal (do Nascimento y Pérez, 2011), o en el análisis bibliométrico en psicología, donde las mujeres conforman solo el $29,45 \%$ (Osca-Lluch y otros, 2013). Sin embargo, esta cifra es semejante a la de otras áreas, como $41 \%$ de mujeres en Ciencia y Tecnología (Mosteiro y Porto, 2014)

La Universidad de Málaga es la que tiene una mayor red de colaboración con otras instituciones en cuanto a la conformación de los tribunales encargados de evaluar las tesis doctorales producidas en T\&I en España.

Este estudio ha permitido identificar algunos patrones en la dirección de las tesis de T\&I y en los tribunales que las han juzgado, facilitando la obtención de una visión global de lo que ocurre en el proceso formativo de los nuevos investigadores en este campo.

\section{REFERENCIAS}

Aixelá, J. F. (2010). Una revisión de la bibliografía sobre traducción e interpretación médica recogida en BITRA (Bibliografía de Interpretación y Traducción). Panace@, 11(32), 151-160.

Alarcón, E. V. (2019). La producción científica española en moda a través de las tesis doctorales. Revista Prisma Social, 24, 209-232.

Baggio, M. A., Rodrigues, M. A., Erdmann, A. L., Figueiredo-Barbieri, M. C., Vieira, M. M. d. S. (2014). Production of nursing thesis and dissertations in Portugal, 2000-2010: a bibliometric study. Texto \& Contexto-Enfermagem, 23(2), 250-260. DOI: 10.1590/010407072014002190012

Boletín Oficial del Estado (1985). Real Decreto 185/1985 del Ministerio de Educación y Ciencia de 16 de febrero por el que se regula la obtención y expedición del título de doctor y de otros estudios posgraduados. $B O E, 1985(41), 3947-3953$.

Boletín Oficial del Estado (1998). Real Decreto 778/1998, de 30 de abril, por el que se regula el tercer ciclo de estudios universitarios, la obtención y expedición del título de Doctor y otros estudios de postgrado. BOE, 1998(104), 14688-14696.

Boletín Oficial del Estado (2001). Ley Orgánica 6/2001, de 21 de diciembre, de Universidades. BOE, 2001(307), 49400-49425.

Boletín Oficial del Estado (2007). Ley Orgánica 4/2007, de 12 de abril, por la que se modifica la Ley Orgánica $6 / 2001$, de 21 de diciembre, de Universidades. $B O E$, 2007(89), 16241-16260.

Boletín Oficial del Estado (2011). Real Decreto 99/2011, de 28 de enero, por el que se regulan las enseñan- 
zas oficiales de doctorado. BOE, 2011(35), 1390913926.

Burnett, P. C. (1999). The supervision of doctoral dissertations using a collaborative cohort model. Counselor education and supervision, 39(1), 46-52.

Fernández-Cano, A., Torralbo, M., Vallejo, M. V. (2008). Revisión y prospectiva de la producción española en tesis doctorales de Pedagogía (1976-2006). Revista de investigación educativa, RIE, 26(1), 191-208.

Casanueva-Roche, C., Escolar-Pérez, B., Larrinaga-González, C. (2007). Red social de Contabilidad en España a partir de los tribunales de tesis. Spanish Journal of Finance and Accounting/Revista Española de Financiación y Contabilidad, 36(136), 707-726.

Castelló-Cogollos, L. (2016). El campo de la Sociología en España (1976-2013). Análisis sociométrico y de redes de las tesis doctorales y sus tribunales. Tesis doctoral, Universitat de Valencia. Valencia.

Comisión Académica del Consejo de Universidades (1996). Resolución 28/1996, de 28 de noviembre, por la que se hace público el acuerdo de 17 de junio de 1996, de la Comisión Académica del Consejo de Universidades, por el que se crean nuevas áreas de conocimiento. (1996, 10 de diciembre). BOE, 297, 36908-36908.

Creswell, J. W., Miller, G. A. (1997). Research Methodologies and the Doctoral Process. New Directions for Higher Education, 99, 33-46.

De Jesus Nascimento, M. (2000). Producción científica brasileña en España: documentación de las tesis doctorales. Ci. Inf, 29(1), 3-13.

Díaz-Campo, J. (2014). La investigación sobre Internet en las facultades de Comunicación españolas. Análisis bibliométrico de tesis doctorales (1997-2012). Documentación de las Ciencias de la Información, 37, 305320. DOI: /10.5209/rev_DCIN.2014.v37.46828

do Nascimento, E. R., Pérez, M. S. T. (2011). Cuestiones de género en las tesis doctorales sobre Enfermería en España. Metas de enfermería, 14(5), 72-76.

Kagra, S. K., Sharma, S. (2014). A reference analysis of doctoral theses in the field Education: An interesting issue for librarians as well as scientometricians or bibliometricians. International Journal of Library and Information Science, 6(3), 22-27. DOI: 10.5897/ IJLIS2013.0410

Kumar, S., Johnson, M., Hardemon, T. (2013). Dissertations at a distance: Students' perceptions of online mentoring in a doctoral program. International Journal of E-Learning \& Distance Education/Revue internationale du e-learning et la formation à distance, 27(1), article_3.

López-Cózar, E. D., Torres-Salinas, D., Jiménez-Contreras, E., Ruiz-Pérez, R. (2006). Análisis bibliométrico y de redes sociales aplicado a las tesis bibliométricas defendidas en España (1976-2002): temas, escuelas científicas y redes académicas. Revista Española de Documentación Científica, 29(4), 493-524.

Maz-Machado, A., Torralbo-Rodríguez, M., Gutiérrez-Arenas, M. P., Morales Sillero, F. (2012a). Citation patter$\mathrm{ns}$ in Educational Science theses at the University of Córdoba. Library Philosophy and Practice (e-journal).

Maz-Machado, A., Bracho-López, R., Torralbo-Rodríguez,
M., Gutiérrez-Arenas, M. P., Jiménez-Fanjul, N., Adamuz-Povedano, N. (2012b). Redes académicas generadas por las tesis doctorales de educación matemática en España. Revista De Investigación Educativa, 30(2), 271-286. https://doi.org/10.6018/rie.30.2.116421

Mosteiro, M. J., Porto, A. M. (2014). Análisis bibliométrico de la producción de tesis doctorales del área de ciencia y tecnología: un análisis por sexo en los espacios escolares. En López, A. J., González, A., \& Aguayo, E. (Eds.): II Xornada Universitaria Galega en Xénero, XUGeX: roles de xénero nun mundo globalizado (págs. 53-57). A Coruña: Universidade da Coruña.

OCDE (1995). La formation a la recherche. Paris: OCDE. Service des Publications.

Olivas-Ávila, J. A., Musi-Lechuga, B. (2010). Producción en tesis doctorales de los profesores funcionarios de Psicología en España más productivos en la Web of Science. Psicothema, 22(917-923).

Olmeda-Gómez, C., Perianes-Rodríguez, A., Ovalle-Perandones, M. A., y De-Moya-Anegón, F. (2009). Colegios visibles: estructuras de coparticipación en tribunales de tesis doctorales de biblioteconomía y documentación en España. El Profesional de la Información, 18(1), 41-49. DOI: 10.3145/epi.2009.ene.06

Olmedilla, A., Abenza, L., Serrano, A., Muñoz, A. M., García-Angulo, A., y Ortega, E. (2017). Estudio bibliométrico de tesis doctorales sobre psicología del deporte. Cuadernos de Psicología del Deporte, 17(2), 121-130.

Ortega, E. Valdivia, P., Olmedilla, A., Martínez, M. T., y Villarejo, D. (2015). Estudio bibliométrico del papel de la mujer en las tesis doctorales de ciencias del deporte. Journal of Sport \& Health Research, 7(2), 139-148.

Osca-Lluch, J., Haba, J., Fonseca, S., Civera, C., y Tortosa, F. (2013). Tesis doctorales españolas sobre análisis bibliométrico en Psicología. Aula Abierta, 41(2), 99-110.

Peña Sánchez, A. R., Jiménez García, M., Ruiz Chico, J., y Pontón Aricha, T. (2019). La producción científica en el "Sector de Hostelería y Turismo": Un análisis bibliométrico de las tesis doctorales españolas en el periodo 1978-2018. Investigaciones Turísticas 18, 71-94. DOI:10.14198/INTURI2019.18.04

Pérez-Escudero, F. (2018). Análisis bibliométrico y webmétrico de los estudios de traducción audiovisual. En E. Cutillas-Orgilés (Ed.), Convergencia y transversalidad en humanidades. Actas de las VII Jornadas de Investigación de la Facultad de Filosofía y Letras de la Universidad de Alicante (pp. 115-122). Alicante: Universidad de Alicante. Facultad de Filosofía y Letras.

Price, D. J. S. (1973). Hacia una ciencia de la ciencia. Barcelona: Ariel.

Ramos-Pardo, F. J., Sánchez-Antolín, P. (2017). Production of educational theory doctoral theses in Spain (2001-2015). Scientometrics, 112(3), 1615-1630. DOI: $10.1007 / \mathrm{s} 11192-017-2435-6$

Repiso, R., Torres-Salinas, D., López-Cózar, E. D. (2013). La investigación científica sobre Cine en España a partir de sus tesis doctorales: Análisis de redes sociales (19782007). Revista ICONO14 Revista Científica de Comunicación y Tecnologías Emergentes, 11(2), 385-404.

Repiso, R., Torres-Salinas, D., López-Cózar, E. D. (2011). Análisis bibliométrico y de redes sociales en tesis doc- 
torales españolas sobre televisión (1976/2007). Comunicar: Revista Científica Iberoamericana de Comunicación y Educación, 37, 151-159.

Santos, J. I., del Olmo, R., Pajares, J. (2006). Estudio de la red de participaciones en tribunales de tesis doctorales de Organización y Gestión de Empresas en España. Comunicación presentada en el X Congreso de Ingeniería de Organización, Valencia.

Seagram, B. C., Gould, J., Pyke, S. W. (1998). An investigation of gender and other variables on time to completion of doctoral degrees. Research in Higher Education, 39(3), 319-335.

Torres-Ramírez, I., Torres-Salinas, D. (2005). Tesis doctorales sobre estudios de las mujeres en España (1976-2002). A propósito de un indicador definitivo en investigación. Revista Española de Documentación Científica, 28(4), 479-499.

Valero-Garcés, C. (2018). Research Tendencies in Translation and Interpreting Studies and Intercultural Com- munication. International Journal of Linquistics, 10(1), 96-107. DOI:10.5296/ijl.v10i1.12750

Velásquez, A., Aguilar, N. (2005). Manual introductorio al análisis de redes sociales: Ejemplos prácticos con UCINET 6.85 y NETDRAW 1.48. México, D.F.: UNAM-Universidad Autónoma de Chapingo.

Wisker, G. (2012). The good supervisor: Supervising postgraduate and undergraduate research for doctoral theses and dissertations. Wakefield, West Yorkshire: Macmillan International Higher Education.

Zanettin, F., Saldanha, G., Harding, S. A. (2015). Sketching landscapes in translation studies: A bibliographic study. Perspectives, 23(2), 161-182.

Zong, Q. J., Shen, H.Z., Yuan, Q.J., Hu, X.-W., Hou, Z.P., Deng, S.G. (2013). Doctoral dissertations of Library and Information Science in China: A co-word analysis. Scientometrics, 94(2), 781-799. DOI:10.1007/ s11192-012-0799-1 